\title{
Slit-lamp evaluation during SARS-CoV-2 pandemic: safety first!
}

\section{Raul E. Ruiz-Lozano ${ }^{1}$ (D) - Lucas A. Garza-Garza ${ }^{1}$ - Jesus A. Cardenas-de la Garza ${ }^{2}$ (D) • Julio C. Hernandez-Camarena ${ }^{1,3}$ (D)}

Received: 20 April 2020 / Revised: 20 April 2020 / Accepted: 26 April 2020 / Published online: 4 May 2020

(C) Springer-Verlag GmbH Germany, part of Springer Nature 2020

Keywords COVID-19 · Slit-lamp evaluation · Safety measures · Physical examination · Coronavirus

Dear Editor,

Unlike other medical specialties, an adequate ophthalmological practice strongly relies on physical examination to reach the diagnosis, which involves close contact with the patient. Moreover, several ophthalmic tools such as the slitlamp, the Goldmann tonometer, gonioscopy and fundus lenses, and the phoropter, among others are required to perform a complete eye evaluation. All of them come in close or direct contact with the patient and hence can favor nosocomial spread of infections. Droplet spread is the main route by which the novel coronavirus (SARS-CoV-2) is transmitted. Studies reveal droplet presence of SARS-CoV-2 on door handles, mobile phones, plastic, stainless steel, and other common contact surfaces [1]. The virus can stay on the surface for 2-3 days, increasing the risk of fomite infectivity [1]. To reduce the spread of SARS-CoV-2, certain steps must be included into the practice:

\section{1) Rescheduling}

a. Elective procedures and patients with no ophthalmic emergency with confirmed or suspected coronavirus disease (COVID-19) should be judiciously rescheduled [2].

Julio C. Hernandez-Camarena

dr.juliohernandez@medicos.tecsalud.mx

1 Tecnologico de Monterrey, Escuela de Medicina y Ciencias de la Salud, Monterrey, Mexico

2 Rheumatology Service, University Hospital "Dr. José Eleuterio González”, Universidad Autónoma de Nuevo León, Monterrey, Nuevo León, Mexico

3 Tecnologico de Monterrey, Instituto de Oftalmologia y Ciencias Visuales, Hospital Zambrano Hellion TecSalud, Av. Batallon de San Patricio No. 112. Col. Real de San Agustin, N.L. CP., 66278 San Pedro Garza García, Mexico b. In March 27, 2020, the American Academy of Ophthalmology enlisted the urgent and emergent procedures such as drainage of abscess, retinal detachment surgery, anterior segment, or cornea laceration repair, among others. The latter includes procedures aiming to resolve potential sight-threatening conditions [3].

2) Before slit-lamp evaluation (SLE)

a. Patients must wear a mask and perform hand washing with soap and water or $80 \%$ ethanol solution (80\% ES) [4].

b. A protective shield on the slit-lamp must be installed and cleaned up after each consultation to reduce droplet transmission.

c. Ophthalmologists must wash hands and wear N95 masks, protective eyewear, and new gloves [5].

d. Contact surfaces from the slit-lamp such as the applanation tonometer and chin and forehead support must be disinfected with $80 \%$ ES [4].

\section{3) During SLE}

a. Avoid talking and instruct the patient to do the same.

b. Touching the patient's eye should be discouraged.

c. Avoid Goldmann applanation tonometry. If strictly necessary, use disposable tips [2].

d. If gonioscopy and fundus evaluation is required, disposable lens use and/or prior disinfection must be performed [5].

4) After SLE

a. Clean the slit-lamp and all the surfaces exposed to the patient with an alcohol wipe.

b. Both the patient and the ophthalmologist, prior glove removal, must wash their hands as mentioned above [2]. 


\section{5) Avoid unnecessary paperwork}

a. A digital patient record is preferred.

Despite most of the ophthalmological practice may be deferred, sometimes it is not possible to distinguish a true emergency without the SLE evaluation. While telemedicine represents a viable option in other medical specialties, ophthalmology represents a real challenge since it relies mainly upon a detailed SLE. Since no vaccine or effective antiviral currently exists, we only have universal public health measures to avoid disease spreading. Meanwhile, ophthalmologists, due to the proximity required to adequately evaluate our patients, are urged to take extreme caution aiming to contain COVID-19.

\section{Compliance with ethical standards}

Conflict of interest The authors declare that they have no conflicts of interest.

\section{References}

1. van Doremalen N, Bushmaker T, Morris DH, Holbrook MG, Gamble A, Williamson BN, Tamin A, Harcourt JL, Thornburg NJ, Gerber SI, Lloyd-Smith JO, de Wit E, Munster VJ (2020) Aerosol and surface stability of SARS-CoV-2 as compared with SARS-CoV1. N Engl J Med 382(16):1564-1567. https://doi.org/10.1056/ NEJMc2004973

2. Lai THT, Tang EWH, Chau SKY, Fung KSC, Li KKW (2020) Stepping up infection control measures in ophthalmology during the novel coronavirus outbreak: an experience from Hong Kong. Graefes Arch Clin Exp Ophthalmol. https://doi.org/10.1007/ s00417-020-04641-8

3. List of urgent and emergent ophthalmic procedures (2020) American Academy of Ophthalmology. https://www.aao.org/headline/list-ofurgent-emergent-ophthalmic-procedures. Accessed 19 Apr 2020

4. Kampf G, Todt D, Pfaender S, Steinmann E (2020) Persistence of coronaviruses on inanimate surfaces and their inactivation with biocidal agents. J Hosp Infect 104(3):246-251. https://doi.org/10.1016/ j.jhin.2020.01.022

5. Khanna RC, Honavar SG (2020) All eyes on coronavirus-what do we need to know as ophthalmologists. Indian J Ophthalmol 68(4): 549-553. https://doi.org/10.4103/ijo.IJO_516_20

Publisher's note Springer Nature remains neutral with regard to jurisdictional claims in published maps and institutional affiliations. 